\title{
Criocirurgia como tratamento de papiloma escamoso em odontopediatria: relato de caso
}

\section{Cryosurgery as squamous papilloma treatment in pediatric dentistry: case report}

\author{
Gustavo Eidt \\ Jorge Ricardo Schmidt Maas** \\ Leo Kraether Neto ${ }^{* * *}$
}

\section{Resumo}

Objetivo: relatar o diagnóstico e tratamento de um possível papiloma escamoso em uma criança por meio da criocirurgia com nitrogênio líquido. Relato de caso: Paciente de 8 anos de idade, sexo masculino, procurou atendimento referindo apresentar "verruga" em borda interna de vermelhão do lábio, próximo à comissura bucal esquerda. Baseado nas características clínicas da lesão, o diagnóstico foi de papiloma escamoso oral. Optou-se pela criocirurgia por se tratar de uma técnica cirúrgica minimamente invasiva e apresentar vantagens teóricas em relação à técnica cirúrgica convencional, principalmente no que diz respeito ao manejo do paciente. Para tal, foram utilizados instrumentos simples e relativamente acessíveis, como garrafa térmica para acondicionamento do gás nitrogênio sob sua forma líquida e swab clínico para aplicação do gás sobre a lesão. Considerações finais: uma semana após o procedimento, houve a eliminação da lesão e uma boa cicatrização do local. O agente de congelamento escolhido e a técnica utilizada provaram-se eficientes como alternativa de tratamento não traumático para o papiloma escamoso.

Palavras-chave: Criocirurgia. Papiloma. Nitrogênio.

\section{Introdução}

Crianças representam uma faixa etária suscetível a diversas patologias características e peculiares a essa fase da vida, além de outras patologias mais "generalistas". O tratamento, se necessário, pode ser limitado por questões físicas e psicológicas específicas da idade do paciente. $\mathrm{O}$ conhecimento e o bom senso do profissional são muito importantes para que a situação possa ser bem conduzida e resolvida.

Técnicas ditas alternativas podem, muitas vezes, trazer diversos benefícios em relação às convencionais. O conhecimento dos princípios de ação dessas técnicas, assim como de suas restrições, permite ao profissional que faça a escolha certa e obtenha o resultado clínico seguro.

Criocirurgia é definida como a destruição de tecido pela aplicação localizada de temperaturas extremamente baixas. Seu uso na medicina tem registro desde 1851, quando o físico inglês James Arnott provou ser possível curar cânceres com o uso de soluções salinas de baixa temperatura em aplicações localizadas. Foi, porém, somente em 1899,

\footnotetext{
Aluno da graduação do curso de Odontologia da Universidade de Santa Cruz do Sul (UNISC), Santa Cruz do Sul, RS, Brasil.

Mestre em Odontopediatria pela Universidade Luterana do Brasil (Ulbra), professor da disciplina de Odontopediatria da UNISC, Santa Cruz do Sul, RS, Brasil.

Mestre em CTBMF pela Universidade Federal de Pelotas (UFPel), doutor em Estomatologia Clínica pela Pontifícia Universidade Católica do Rio Grande do Sul (PUC-RS), coordenador do Projeto Diagnóstico Bucal da UNISC, Santa Cruz do Sul, RS, Brasil.
} 
quando o americano Campbell White reportou o uso de agentes refrigerantes como o ar líquido, que a criocirurgia obteve uma eficácia satisfatória como meio de tratamento para lesões dermatológicas diversas $^{1}$. Sua aplicação hoje se estende de tumores hepáticos a ósseos ${ }^{2,3}$.

Em muitos casos, a criocirurgia é uma alternativa para pacientes aos quais seja contraindicada a técnica cirúrgica convencional, pois possui várias vantagens, como ausência de sangramento, baixo desconforto (anestésicos injetáveis geralmente não são utilizados) e adequado processo de cicatrização. Além disso, o risco de infecções é bastante reduzido, na medida em que o tecido conjuntivo não é imediatamente exposto ${ }^{4,5}$.

O papiloma escamoso é uma proliferação benigna do epitélio escamoso estratificado, que resulta em uma massa papilar ou verrucosa. Presumivelmente, a lesão é induzida pelo papilomavírus humano (HPV). Existem pelo menos 24 tipos de HPV associados com lesões de cabeça e pescoço. Aparentemente, a virulência e a taxa de infectuosidade nesse tipo de lesão são extremamente baixas, diferentemente de outras lesões induzidas por $\mathrm{HPV}^{6}$.

Existem diversos tipos de papilomas, mas os que ocorrem na boca são quase sempre escamosos. O papiloma escamoso bucal é uma denominação genérica, usada para incluir crescimentos papilares e verrucosos nessa região. É a lesão papilar mais comum da mucosa bucal (incluindo a parte do vermelhão do lábio), constituindo aproximadamente $3 \%$ das lesões de boca ${ }^{7}$.

Reportado, pela primeira vez, como "verruga gengival" por Tomes, em 1848, o papiloma oral, tipicamente, tem o aspecto de um nódulo exofítico mole, pedunculado com numerosas projeções na sua superfície, as quais podem estar cobertas com generosa quantidade de queratina, fazendo que apresente coloração esbranquiçada (Figura 1). Os locais de predileção para essa lesão são a língua, os lábios e o palato, mas qualquer superfície bucal pode ser afetada. O papiloma é uma lesão geralmente solitária. Seu crescimento é rápido, e seu tamanho máximo é normalmente inferior a $0,5 \mathrm{~cm}$ de diâmetro, podendo variar. As projeções podem ser pontudas ou rombudas ${ }^{6,8,9}$.

Os subtipos HPV-6 e HPV-11 são os mais comumente isolados de papilomas orais. É interessante ressaltar que essas viroses não estão entre as da família HPV supostamente responsáveis pela malignização de lesões pré-cancerígenas. A experiência clínica demonstra que papilomas orais não tendem a malignizar e raramente tendem a aumentar de tamanho ou desaparecer por conta ${ }^{8}$.

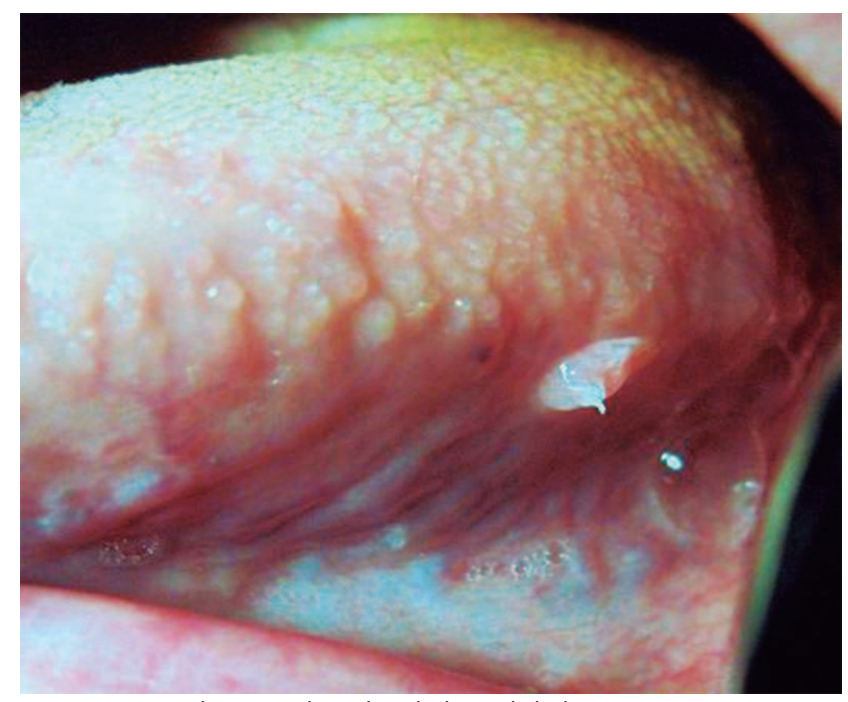

Figura 1 - Papiloma oral em borda lateral de língua (acervo do projeto Diagnóstico Bucal da UNISC)

A verruga vulgar é, também, uma hiperplasia focal do epitélio escamoso estratificado que está relacionada, principalmente, aos subtipos virais HPV2 e HPV-4, entre outros. Comumente encontrada nas mãos de crianças, é contagiosa e acomete a pele, mas pode também atingir a mucosa oral, principalmente por autoinoculação. Quando isso acontece, a localização preferencial é borda do vermelhão do lábio, a mucosa labial ou a ponta da língua. Clinicamente, a lesão é indistinguível do papiloma ${ }^{10}$.

Frequentemente, clínicos consideram que o papiloma oral é uma lesão que se manifesta na infância, mas o diagnóstico, de fato, é feito, via de regra, em pessoas entre 30 e 50 anos de idade. Um dos aspectos fascinantes do papiloma oral é que ele se comporta de modo diferente de outros papilomas. Por exemplo, papilomatoses laríngeas, apesar de clinicamente idênticas às orais, apresentam índice de recidiva bastante alto, são geralmente múltiplas e continuam proliferando com o tempo, podendo, inclusive, levar à morte por asfixia ${ }^{6,8}$.

O tratamento para o papiloma oral é conservador, sendo necessária a remoção completa da lesão. Margem de segurança não é obrigatória. Lesões não tratadas normalmente não mudam com o tempo. A excisão cirúrgica conservadora é uma boa escolha, sendo também indicadas a destruição por laser de $\mathrm{CO}_{2}$, a eletrocauterização e a criocirurgia. Porém, deve-se ter em mente que, se tais técnicas forem utilizadas, o diagnóstico definitivo por biópsia é excluído, a não ser que uma porção da lesão seja primeiramente removida para análise histológica ${ }^{8}$.

O presente trabalho tem como objetivo relatar o diagnóstico e tratamento de um possível papiloma escamoso em uma criança por meio da criocirurgia com nitrogênio líquido. 


\section{Relato de caso}

Paciente faioderma, 8 anos de idade, sexo masculino, compareceu ao Projeto Diagnóstico Bucal da UNISC relatando apresentar "verruga" em borda interna de vermelhão do lábio, próximo à comissura bucal esquerda (Figura 2).

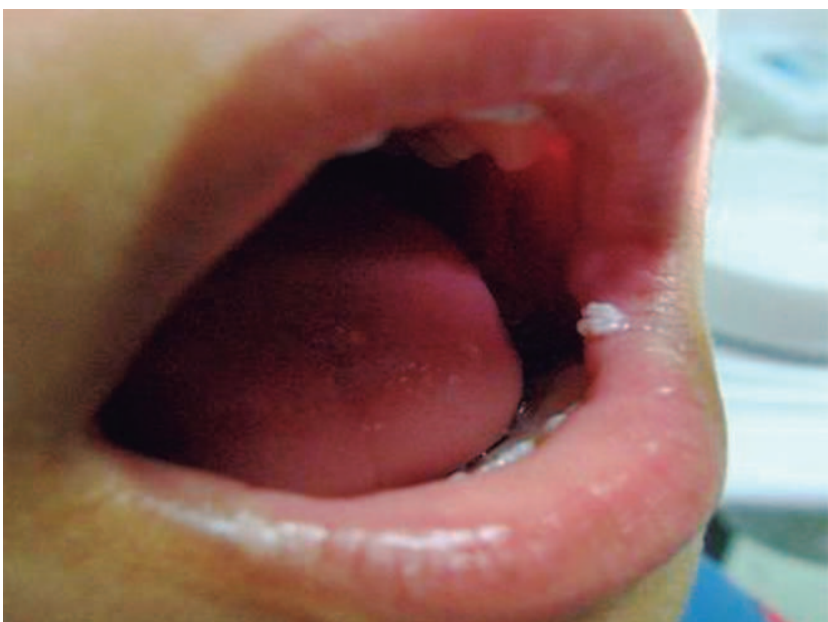

Figura 2 - Aspecto clínico inicial da lesão em região interna de comissura bucal esquerda (acervo do projeto Diagnóstico Bucal da UNISC)

O diagnóstico clínico foi de papiloma escamoso oral ou verruga vulgar, provavelmente proveniente de autoinoculação por lesões prévias em pele das mãos. O tratamento para ambas as lesões seria a excisão cirúrgica conservadora, porém, em seu lugar, optou-se pela técnica de criocirurgia, principalmente pelo fato de o paciente apresentar perfil pouco colaborador para que a técnica cirúrgica convencional fosse empregada.

Para uma melhor conservação do gás em seu estado líquido, optou-se pelo uso de garrafa térmica de aço inox. É importante que a transferência do líquido do reservatório para o meio de transporte seja feita o mais próximo possível do atendimento clínico e que o recipiente térmico de transporte seja sempre apenas parcialmente fechado, buscando evitar o risco de explosão pela descompressão do gá ${ }^{11}$.

Para aplicação do agente de congelamento, optou-se pelo uso de swab clínico estéril. Um swab, com prolongamento, foi utilizado diretamente para imersão no nitrogênio líquido e aplicação sobre a lesão, e outro swab, sem prolongamento, foi empregado para manter a pressão após a aplicação do frio, melhorando, assim, a troca térmica para acelerar o ciclo de congelamento e descongelamento do tecido.

As aplicações de frio foram intermitentes, com duração aproximada de 2 segundos cada. Os intervalos entre aplicações, quando o segundo swab era empregado, tinham duração equivalente. Na mesma sessão clínica, foram feitas 30 aplicações, tendo, após as 15 primeiras aplicações de frio, havido uma parada de aproximadamente 10 minutos para monitoramento do edema local, antes de se proceder às
15 aplicações restantes. Esse protocolo não é rígido e pode variar desde que os princípios do procedimento sejam respeitados. Tempos de congelamento totais de 1-2 minutos são sugeridos para a maioria das lesões orais benignas de tecido mole. ${ }^{4}$

É interessante observar que o aspecto da lesão após o término das aplicações permaneceu inalterado, a não ser pela presença de leve edema local. Sete dias após o procedimento, o paciente retornou para reavaliação clínica. $\mathrm{O}$ aspecto cicatricial da região onde antes se encontrava a lesão era satisfatório (Figura 3). Além disso, o paciente relatou ter apresentado pouco desconforto durante e após a criocirurgia. A lesão esfoliou no segundo dia decorrida a aplicação do frio e não houve sangramento nem exposição direta do tecido conjuntivo.

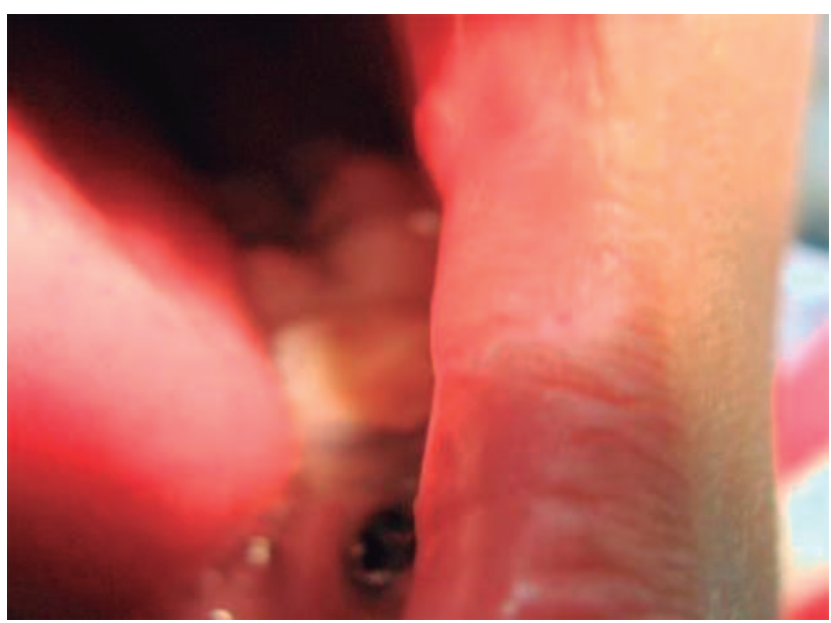

Figura 3 - Aspecto cicatricial da região uma semana após o procedimento da criocirurgia. (acervo do projeto Diagnóstico Bucal da UNISC)

\section{Discussão}

Os princípios do funcionamento da criocirurgia são simples, porém interagem de maneira complexa. Basicamente, à medida que a temperatura no tecido fica abaixo da de congelamento da água, formam-se cristais de gelo na matriz extracelular. Isso cria um ambiente hiperosmótico extracelular que sequestra água do interior das células adjacentes, desidratando-as. Quando a temperatura no tecido fica inferior a $-20^{\circ} \mathrm{C}$, ocorre a formação de cristais de gelo também no interior das células. A seguir, dá-se o derretimento dos cristais extracelulares com a consequente passagem de água para o interior das células previamente desidratadas e injuriadas. Com a repetição desse ciclo, ocorre o rompimento da membrana celular ${ }^{4,12}$.

O frio extremo também afeta a microcirculação sanguínea. Ocorre diminuição do fluxo sanguíneo, trombose progressiva com perda do suprimento local de sangue e consequente necrose isquêmica. $\mathrm{Na}$ sequência, ocorre uma vasodilatação local compensatória que pode perdurar até 15 dias. O processo inflamatório subsequente acarreta, sobretudo, ede- 
ma local e esfoliação das células atingidas, contribuindo para o resultado clínico esperado pela técnica ${ }^{13}$.

A escolha do agente de congelamento é importante para o sucesso do procedimento. Sabe-se que temperaturas entre $-20^{\circ} \mathrm{C} \mathrm{e}-30^{\circ} \mathrm{C}$ devem ser alcançadas no tecido para que a destruição celular ocorra. $\mathrm{O}$ agente mais comumente utilizado para isso é o nitrogênio líquido. Trata-se do gás $\mathrm{N}_{2}$ em estado líquido a uma temperatura de $-196^{\circ} \mathrm{C}$, o qual é obtido industrialmente em larga escala por meio da destilação fracionada do ar. Apesar do risco de danos teciduais em caso de contato com esse agente, $o$ seu manuseio é simples e o produto pode ser acondicionado em garrafas térmicas semiabertas, onde ocorre uma lenta evaporação e liberação do gás ${ }^{11}$.

$\mathrm{O}$ agente de congelamento deve ser aplicado somente sobre a área de tecido a ser destruído. $\mathrm{O}$ tamanho da ponta ativa de aplicação e a consequente massa de tecido congelado devem ser determinados de acordo com o tamanho da base da lesão e a sua profundidade. A duração do período de congelamento, dada pelo tempo de aplicação do frio sobre o tecido em um ciclo, deve ser mínima, de modo a proporcionar o congelamento necessário do tecido e não se estender demais, para evitar dor desnecessária ao paciente. O número de ciclos de congelamento está diretamente relacionado aos danos sofridos pelas células expostas e já previamente enfraquecidas, aumentando-se o tamanho dos cristais de gelo intracelulares de acordo com a repetição dos ciclos ${ }^{13}$.

A natureza do tecido exposto também é determinante, pois a suscetibilidade a baixas temperaturas varia conforme o tipo de célula presente no tecido. Por exemplo, células musculares e melanócitos são mais sensíveis ao congelamento do que células cartilaginosas e queratinócitos. Células cancerosas aparentam ser mais sensíveis do que células normais $^{6,11}$.

Alguns detalhes biológicos, como o papel da criocirurgia no sistema imunológico, ainda não são completamente entendidos. Além disso, a técnica apresenta limitações quanto ao domínio da profundidade de danos ao tecido e da sua resposta. Apesar disso, a prática da criocirurgia já está bem consolidada e consiste em uma alternativa segura para remoção de diversas lesões no corpo. Com o avanço das pesquisas e do conhecimento sobre a técnica, sua aplicação clínica tende a tornar-se mais indicada e abrangente ${ }^{13,14}$.

O manejo do paciente é muito importante para o sucesso de qualquer abordagem odontológica, principalmente em pediatria. Quando pode se optar por uma técnica minimamente invasiva, esta deve ser a primeira escolha.

A correta indicação do tratamento por criocirurgia depende de vários fatores. Porém, é imprescindível que o diagnóstico das lesões seja feito clinica- mente e que não seja duvidoso. A biópsia deve ser sempre a escolha caso isso não seja possível. Quando corretamente empregada, a técnica da criocirurgia pode apresentar diversas vantagens na remoção de papilomas, fibromas, hiperplasias inflamatórias pequenas, entre outras lesões de comum acometimento oral.

\section{Considerações finais}

O resultado clínico obtido pelo emprego da técnica de criocirurgia para remoção de papiloma escamoso em paciente de 8 anos de idade foi satisfatório. A técnica provou ser simples e segura. Além disso, o desconforto para o paciente foi mínimo e a cicatrização foi rápida e efetiva. Apesar de ser considerada uma técnica alternativa, a criocirurgia provou ser viável para o ambiente ambulatorial do cirurgião-dentista.

\section{Abstract}

Objective: to report the diagnosis and treatment of a possible squamous papilloma in a child using cryosurgery with liquid nitrogen. Case report: an 8-year-old male patient sought assistance claiming to present a "wart" on the inner edge of the lip vermilion, near the left oral commissure. Based on the clinical features of the lesion the diagnosis was an oral squamous papilloma. Cryosurgery was chosen for treatment because of his minimally invasive surgical characteristics and theoretical advantages relating to the conventional surgical technique, especially with regards to patient management. Hence, the instruments used for the technique were very simple and relatively inexpensive, such as a thermos bottle for conditioning of the liquid nitrogen, and a clinical swab to be applied on the lesion. Final considerations: one week after the procedure we found the elimination of the lesion and a good local healing. The freezing agent and the chosen technique have proved to be effective as an alternative of non-traumatic treatment for squamous papilloma.

Keywords: Cryosurgery. Papilloma. Nitrogen.

\section{Referências}

1. Cooper SM, Dawber RPR. The history of cryosurgery. J Royal Soc 2001; 94(4):196-201.

2. Malawer MM, Bickels J, Meller I, Buch RG, Henshaw RM, Kollender Y. Cryosurgery in the treatment of giant cell tumor: a long term follow up study [online]. Clinical Orthopaedics \& Related Research 1999; 359:176-88. Disponível em URL: http://www.sarcoma.org/html_articles/cryo\%20gct. htm.

3. Adam R, Akpinar E, Johann M, Kunstlinger F, Majno P, Bismuth $\mathrm{H}$. Place of cryosurgery in the treatment of malingnant liver tumors. An of Surgery 1997; 225(1):39-50. 
4. Farah CS, Savage NW. Cryotherapy for treatment of oral lesions. Aust Dent J 2006; 51:(1):2-5.

5. Carneiro TE, Marinho SA, Verli FD, Mesquita AM, Lima NL, Miranda JL. Oral squamous papilloma: clinical, histological and immunohistochemical analyses. J Oral Sci 2009; 51(3):367-72.

6. Neville BW, Damm DD, Allen CM, Bouquot JE. Patologia oral \& maxilofacial. 3. ed. Rio de Janeiro: Elsevier Saunders; 2009.

7. Regezi JA, Sciubba JJ. Patologia bucal. Correlações clínico-patológicas. 3. ed. Rio de Janeiro: Guanabara Koogan; 1999. p. $144-57$.

8. Bouquot JE, Wrobleski GJ. Papillary (pebbled) masses of the oral mucosa: more than simple papillomas. Pract Periodontics Aesthed Dent 1996; 8(6):533-43. Disponível em URL: http://www.maxillofacialcenter.com/papillary96.html.

9. Yiu W, Basco MT, Aruny JE, Cheng SWK, Sumpio BE. Cryosurgery: a review. Int J Angiol 2007; 16(1):1-6.

10. Reichert PA, Philipsen HP. Patologia Bucal. Porto Alegre: Artes Médicas Sul; 2000.

11. Allington HV. Liquid nitrogen in the treatment of skin diseases. California Medicine 1950; 72:153-5.

12. International Society of Cryosurgery (ISC). Disponível em URL: http://www.societyofcryosurgery.org/.

13. Yu C-H, Chen H-M, Chang C-C, Hung H-Y, Hsiao C-K, Chiang C-P. Cotton-swab cryotherapy for oral leukoplakia. Head Neck 2009; 31:983-8.

14. Smith KJ, Skelton H. Molluscum contagiosum: recent advances in pathogenic mechanisms and new therapies. Am J Clin Dermatol 2002; 3(8):535-45.

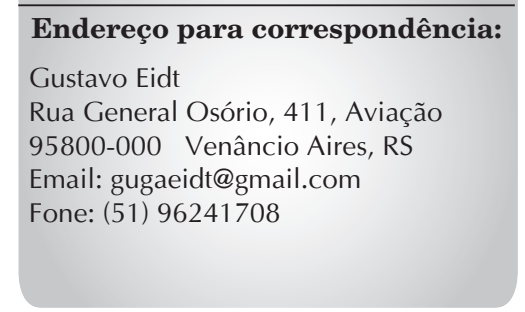

Recebido: 13/08/2013. Aceito: 03/10/2013. 